\title{
Design and Realization of the Accelerometer based Transportation System
}

\author{
Deepak Punetha \\ Electronics Engineering Dept. \\ PEC University of Technology \\ Chandigarh, India
}

\author{
Deepak Kumar \\ Electronics Engineering Dept. \\ PEC University of Technology \\ Chandigarh, India
}

\author{
Vartika Mehta \\ Electronics Engineering Dept. \\ Dehradun Institute of Tech. \\ Dehradun, India
}

\begin{abstract}
An accident is a deviation from expected behavior of event that adversely affects the property, living body or persons and the environment. Security in vehicle to vehicle communication or travelling is primary concern for everyone. The work presented in this article documents the designing of an accident detection system. The accident detection system design informs the police control room or any other emergency calling system about the accident. An accelerometer sensor has been used to detect abrupt change in g-forces in the vehicle due to accident. When the range of gforces comes under the accident severity, then the microcontroller activates the GSM modem to send a prestored SMS to a predefined phone number. Also a buzzer is switched on. The product design was tested in various conditions. The test result confirms the stability and reliability of the system.
\end{abstract}

\section{Keywords}

Accelerometer; automatic emergency calling system; Accident detection; abrupt change in g-forces detector; GSM Modem.

\section{INTRODUCTION}

Vehicle accident is a very big problem for India and other countries too. This Transportation system aims at providing help to the injured in road accident as fast as possible, thereby this system can increase the chances of the survival accident victim. For an accident victim it is very difficult to alert the police control room or the family members about an accident. The main motive behind this project is to help the accident victims by providing them instant help by SOS services. By using this system, the number of deaths can be reduced. This transportation system is the cost effective system, which could be very helpful to the every-one.

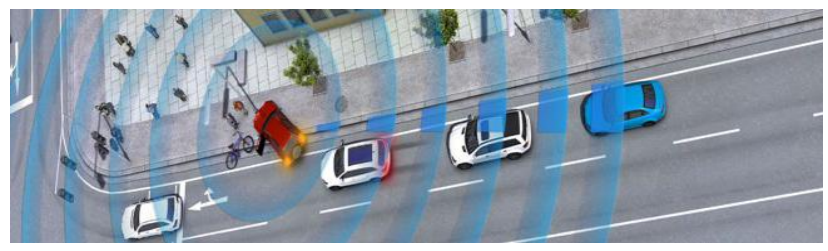

Fig 1: Illustration of Car Accident Alert System [1]

The article reports the motivation and the problem in the transportation system. After the problem was defined a details study of related work done was carried out. During the study of related work a lot of scope was seen to improve the present technology in accident alert system. After the problem was defined completely, the hardware implementation of the designed product was thought off. The hardware was designed and tested in various conditions.

\section{MOTIVATION}

Road accident records in India say that $16 \%$ of the world's road accident deaths happen in India only, while India has only $1 \%$ of the world's road vehicles [2]. In Mumbai's 26/11 bomb blast 195 people killed, but if we checked about the death victim in road accident in India, then we find that 250 people die in road accidents in India every day. If we discuss the data, then Mumbai's 26/11 type of bomb blast happens each and every day in India. Now, it is a big question to every buddy that which one is a big killer, Road accident or Mumbai's 26/11 bomb blast. Other countries are also facing these type of problems [3]. Some statistics shows that the main cause of death of the peoples in road accident is the delay in providing emergency services [4]. Having known about such type of statistics, it was thought off to make a detection system which is able to give the information or alert about the accident occurred to police control room or family member of the accident victim [5]. This vehicle detection and alert system may help the human by saving the life of accident victims.

\section{PROBLEM DEFINITION}

Various enquiry reports of rod accidents were studied and it was clear that the major reason for road accident death was the delay in providing help to the accident victims. The Accelerometer based transportation system proposed would inform the police control room or accident victim's family members about the accident instantaneously, so that help to the injured in road accident could be delivered as soon as possible. The medical emergency care unit would dispatch to the accident location without any delay, thereby we can increase the victim's survival chances.

\section{RELATED WORK}

In the field of transportation system, car to car communication area is very popular area of research. Many systems deal with the accident detection [6]. In such type of detection system vibration sensor play a vital role. We can detect the accident by abrupt vibration in the vehicle at the time of accident. The major problem in existing system is misfiring of the alert system. The airbag system is used to save the life of accident victim. The airbag systems have explosive firing, and have high contact forces resulting in head and neck injuries. Front bumper sensors can be used for both airbag deployment and pedestrian protection systems [7]. Vehicular Ad-Hoc 
Networks are also used as the accident detection system. Since the vehicles can be have very sporadic mobility. Consequently, for the maintaining high performance in the network system, we have to construct the topology, physical layer and the routing protocol of the network system [8]. This system is known to face problems in judging whether or not an accident has occurred [5]. The Accelerometer based transportation system could be helpful in sensing the change in g- forces and also provide automation emergency calling system. This system may help to save the life of accident victim by providing information about accident detection or crash detection through GSM module.

\section{HARDWARE IMPLIMENTATION 5.1 Overview}

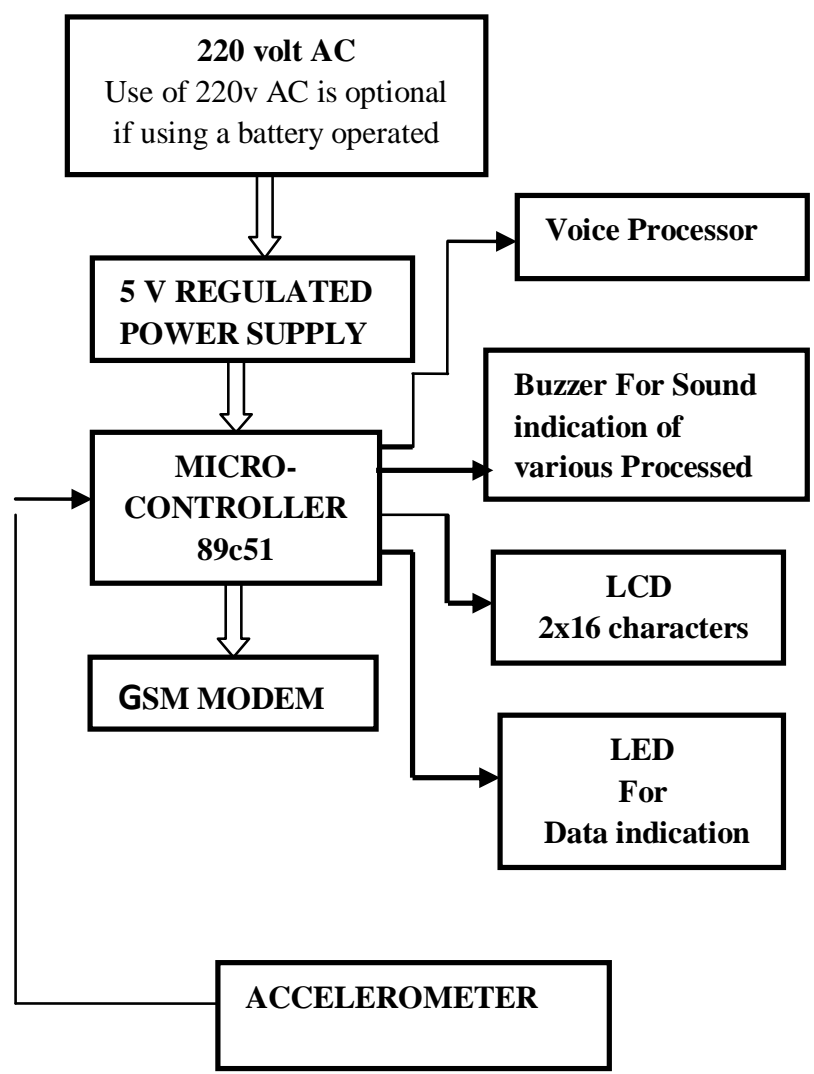

Fig 2: Accelerometer based Transportation System Block Diagram

\subsection{Automatic Emergency calling system}

For automatic emergency calling system we used GSM modem. The GSM network is used to provide communication from one place to another [9]. Using the GSM module consists of a Mobile Station (ME and SIM) [10]. The commands used to provide communication were AT commands. The AT commands specify the GSM technology and are related to SMS service [11]. If an accident happens, the GSM modem is used as the automatic emergency calling system [12]. When the accelerometer sensor senses the change in g-forces in the vehicle at the time of accident, we set the flag of the microcontroller unit (MCU). MCU set the pin of LED for data indication. If LED is ON that means Vibration sensor has detected the crash. The MCU sends a command to the GSM modem to send a pre-stored massage to a predefined telephone number, which may be a SOS number.
Also the real time clock is stopped at the instant of the accident and the time is displayed on a LCD.

\subsection{Sensor module}

Table 1. Thresholds g-forces for Accident Detection

\begin{tabular}{|c|c|}
\hline Accident Severity & $\begin{array}{c}\text { Actual Maximum G } \\
\text { Range Represented [13] }\end{array}$ \\
\hline No Accident & $0-4 \mathrm{~g}$ \\
\hline Mild Accident & $4-20 \mathrm{~g}$ \\
\hline Medium Accident & $20-40 \mathrm{~g}$ \\
\hline Severe Accident & $40+\mathrm{g}$ \\
\hline
\end{tabular}

The accelerometer sensor is based on the detection of the $\mathrm{g}$ forces in the system [13]. This sensor senses the change in the g-forces in the object. The value of the g-forces tells about the nature of the collision or crash [14]. This is the cost effective sensor, which has very high sensitivity [10]. Accelerometers are needed for providing a measure of vehicle body movement for use in electronically controlled suspension systems, passive restraint (eg. air bag) systems and advanced braking systems. These accelerometers must accurately measure low level accelerations from d.c. (or close to d.c.) up to around $50 \mathrm{~Hz}$ or above depending on application. To do this the accelerometer must have a high sensitivity, the characteristics must be very stable in an automotive environment and the response must be essentially flat over the measurement frequency range. Additionally, the accelerometers required for crash sensing will probably require some form of self-diagnostics for early detection of sensor failure. We set a threshold value for the crash or accident detection to the microcontroller, from which we can obtain the result. The accelerometer sensor module is the primary factor for vehicle accident detection and alert system. After sensing the change in the g-forces we use the automatic emergency calling system

\section{WORKING}

\subsection{Accelerometer based Transportation System(ATS)}

The flow chart of the ATS is given in the figure 3. When the vehicle detects abrupt change in the g-forces with the help of accelerometer sensor, which set the flag bit of microcontroller $89 \mathrm{c} 51$ as soon as accident is detected [15]. We set the effective sensitive value for accelerometer sensor, in which accident or crash can be detected [13]. When microcontroller detects the accident or set bit through accelerometer sensor, it further give the display in LCD that accident had occurred and indicate the accident through LED. Simultaneously Microcontroller activates the GSM module [10], which have a manually saved phone number of police control room or family member of accident victim, sends a pre stored SMS to that number [9]. This system is called as Automatic Emergency calling system [11]. We also have an alarm system in the location, which activates when crash is detected and sounds through the buzzer.

The ATS system describes a design of effective alarm system that can monitor a vehicle/ car condition while travelling. This system is designed to inform about an accident or crash that had occurred, to the police control room or the family members of the travelling persons. ATS system uses a piezoelectric sensor which can detect the abrupt vibration when an accident or crash had occurred. This sends a signal to microcontroller [10]. A GSM modem is interfaced with the 
microcontroller unit (MCU). The GSM modem sends an SMS to the predefined mobile number and informs about the accident.

ATS is built around the AT89C51 microcontroller from Atmel. This microcontroller provides all the functionality of the SMS alert system [15]. It also takes care of filtering of the signals at the inputs. Only after an input remains unchanged for 30 milliseconds, is the new signal level is passed on for processing by the microcontroller program. This time can be varied by adopting small change in the source code. The uniqueness of the ATS system is, not only alerting the neighbors by its siren, but also it sends an caution SMS to mobile numbers. This numbers can be changed by programming in microcontroller [16].

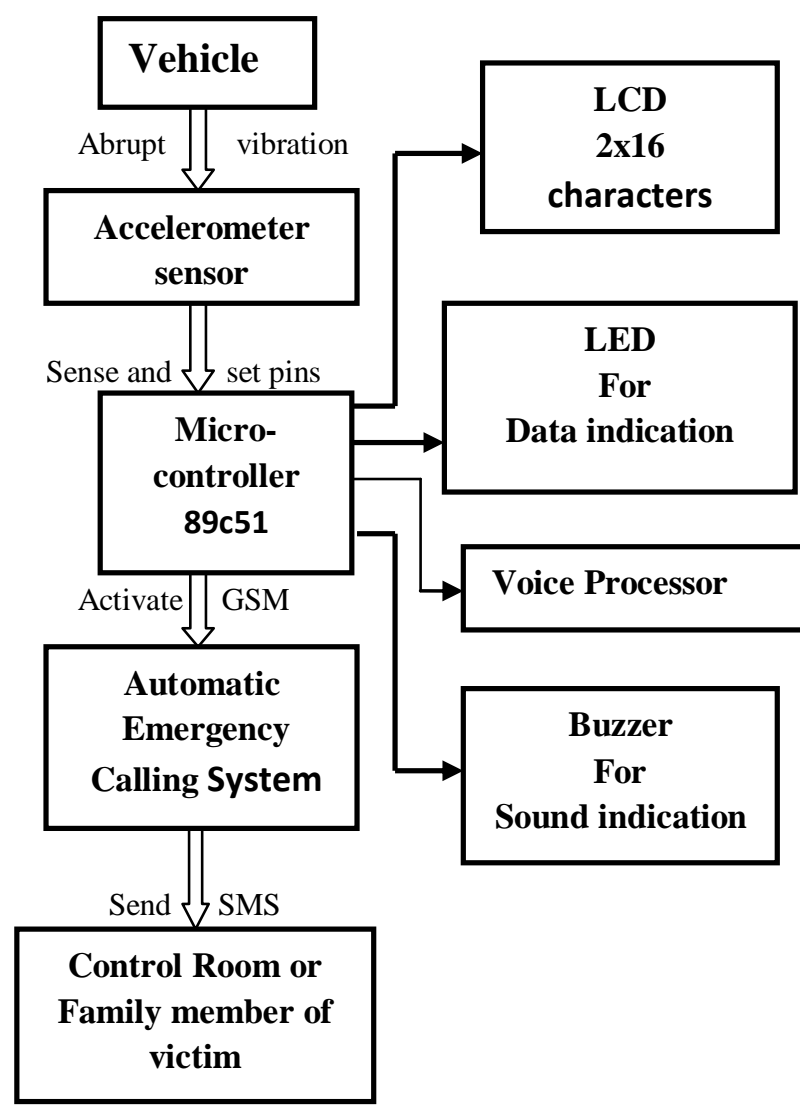

\subsection{System Setup}

Fig 3: Procedure

The ATS system is able to detect the crash or accident detection by using settle the value of sensitivity of accelerometer sensor, which can be measure in the terms of $g$ forces. The interfacing of microcontroller with the LCD and GSM module is shown in figure 4.

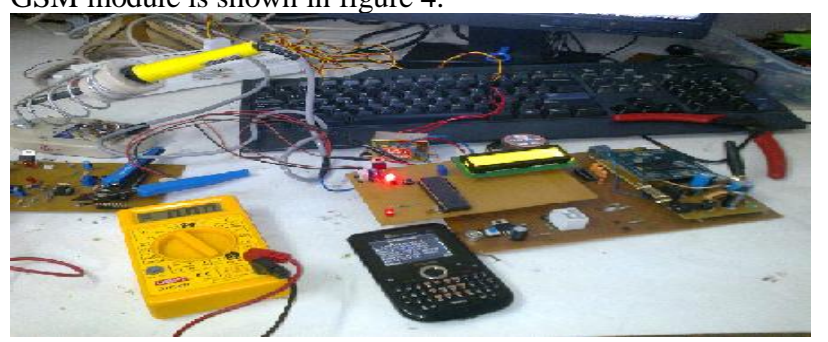

Fig 4: ATS Setup at Lab

\section{EXPERIMENTAL RESULTS}

Due to the influence of the earth's gravitational field, changes in the vehicle attitude will result in apparent accelerations which must be distinguished from genuine vehicle accelerations. Also, accelerometers for measuring horizontal and vertical movement will experience different forces in their rest positions, and so generally their outputs must be set differently. Unless accelerometers are mounted with high positional accuracy it may be necessary to perform a final sensor calibration after they have been mounted onvehicle. For convenience this may be performed by measuring the sensor outputs when the vehicle is horizontal and also when it is positioned on a sloping ramp. Large amplitude accelerations outside the measurement frequency range should ideally be removed by mechanical filtering, most conveniently in the final sensor assembly and packaging. If this is not done then slight rectification of the signal might occur, due to asymmetrical damping and over range protection, resulting in an apparent shift in the measurement signal.

The product of the research documented in this article was tested by placing it on a scrap car. The car was collided with a concrete wall with varying speeds. The speeds could be universal till $40 \mathrm{~km} / \mathrm{hr}$ due to some limitations. The sensitivity of the accelerometer sensor is calculated in the terms of the gforces, which is calculate at the time of collision. The accident is detected by presence of mechanical vibration in the vehicle at the time of accident. The threshold value of the sensitivity can be set.

The crash test report is given in table 2 .

Table 2. Results of Crash Test

\begin{tabular}{|c|c|c|c|c|}
\hline $\begin{array}{c}\text { S.N } \\
\text { O. }\end{array}$ & $\begin{array}{c}\text { g- } \\
\text { forces }\end{array}$ & $\begin{array}{c}\text { Accident } \\
\text { Severity }\end{array}$ & $\begin{array}{c}\text { LCD } \\
\text { Display }\end{array}$ & $\begin{array}{c}\text { Massage } \\
\text { Send }\end{array}$ \\
\hline 1. & 0 & No Accident & Safe & NO \\
\hline 2. & 5 & Mild Accident & Accident & NO \\
\hline 3. & 10 & Mild Accident & Accident & NO \\
\hline 4. & 20 & Mild Accident & Accident & NO \\
\hline 5. & 30 & Medium Accident & Accident & YES \\
\hline 6. & 40 & Medium Accident & Accident & YES \\
\hline 7. & 50 & Severe Accident & Accident & YES \\
\hline
\end{tabular}

Evident from table 2 that whenever an accident with considerable impact takes place, a massage is sent through emergency calling system. The SMS received at the receiver numbers as follows:

"IT IS AN EMERGENCY. A WHITE CAR WITH PLATE NUMBER: XYZ HAS BEEN CRASHED. THIS CAR IS BELONGING TO Mr. DEEPAK PUNETHA. PLEASE URGENT TRY TO HELP ME." 


\section{FUTURE WORK}

This system can be interfaced with vehicle airbag system that prevents vehicle occupants from striking interior objects such as steering wheel or window. This can be developed by interfacing a camera to the controller Module that photograph of the accident spot that makes the tracking easier. The vehicle which is met an accident can be identified by using tracking technology without any delay [16]. The immediate medication will be provided to the accident victims in remote areas. It can also be implemented using accelerometer in series connections in place of piezo-electric vibration sensor. The accelerometer is used to calculate the motion of the object with respect to the axis. Perfect crash detection can be done by using the resultant axis of more than one accelerometer in series. That system could be helpful in determining the crash or accident detection. Sensors mounted on an automobile front bumper are ideal for both frontal airbags and pedestrian protection systems.

\section{CONCLUSION}

ATS can play a vital role in the field of road accidents. By using automatic calling system, this ATS system provide help to the injured person in road accidents as fast as possible, thereby we can increase the victim's survival chances. If the vehicle companies tie-up with ATS system then the transmitting system could be used in the vehicle. For old vehicle, there should be an option to setup the ATS system in it. To minimize the deaths and the severe conditions due to accidents the GSM technologies are used where the immediate action would be taken by the ambulance / police service which might reduces the severity.

\section{REFERENCES}

[1] Car 2 Car Communication Consortium. (2010, May) Car 2 Car Communication Consortium Website. [Online]. http://www.car-to-car.org/

[2] Car Accident Statistics from around the world, Available: www.articlesbase.com

[3] William M Evanco, "The Impact of Rapid Incident Detection on Freeway Accident Fatalities", MS-IVHS.

[4] Statistics of Accidents in India, Available: http://www.nitawriter.wordpress.com/2008/

[5] David A. Whitney and Joseph J Pisano TASC, Inc., Reading, Massachusetts, "Auto Alert: Automated Acoustic Detection of Incidents", IDEA project.
[6] A Ericsson White Paper,"Communication and Information Services for National Security and Public Safety", Ericsson Microwave System AB [Online], Available: http://www.ericsson.com/products/white_papers_pdf/295 2_nsps_a.pdf

[7] Andy Kim, " Smart Crash Detection Using Distributed Sensors", Sponsored by BMW AG.

[8] Scott J. Weiner and Gunar Schirner," Feasibility of a 802.11 VANET Based Car Accident System" Northeastern University, Boston, Technical Report 2010.

[9] Chris Thompson, Jules White, Brian Dougherty, Adam Albright and Douglas C.Schmidt, Vanderbilt University, Nashville, TN USA," Using Smart phones to Detect Car Accidents and Provide Situational Awareness to Emergency Responders".

[10] Datasheets of microcontroller AT89C51, piezo-electric vibration sensor, GSM module, 16*2 LCD

[11] Martin Backstrom, Andreas Hardrup, Tomas Nylander, Jari Vikberg and Peter Ohman, "Mobile Home-GSM Services over Wireless LAN", Ericsson Review, No.2, 2005.

[12] CGALIES, "Report on Implementation Issues Related to Access to detection Information by emergency Services(E112) in the European Union", CGALIES, Final Report.

[13] IEEE Technical Committee for Sensor Technology. (2009, October) The IEEE P1451.6 Project. [Online]. http://grouper.ieee.org/groups/1451/6/index.htm

[14] General Motors, "New Car Assessment Program Frontal Impact Test," MGA Research Corporation, Burlington, Crash Test Report NCAP-MGA-2006-012, 2006.

[15] Rainer D" omer et al., "System-on-Chip Environment: A SpecCBased Framework for Heterogeneous MPSoC Design," EURASIP Journal on embedded Systems, vol. 2008, no. Article ID 647953, p. 13, June 2008.

[16] The Intsitute of Electrical and Electronics Engineers. (2009, October) The IEEE 802.11 Standard. [Online]. http://standards.ieee.org/getieee802/index.html 